\title{
FRACTIONAL INTEGRALS OF GENERALISED FUNCTIONS
}

\author{
A. ERDÉLYI
}

(Received 6 July 1970)

Communicated by B. Mond

\section{1}

The concept of integrals of fractional order of a function $f$, defined by

$$
I^{\alpha} f(x)=\frac{1}{\Gamma(\alpha)} \int_{0}^{x}(x-u)^{\alpha-1} f(u) d u
$$

if $\operatorname{Re} \alpha>0$, can be extended to generalised functions in the framework of the theory of convolution of distributions. The resulting theory [2, Chap. I §5.5] is very satisfactory for many purposes but there are circumstances in which it is not suitable. Such circumstances arise in particular if it is necessary to multiply, before or after integration, by non-integral powers of the variable. Pointwise multiplication by fractional powers of the independent variable does not make sense in the theory of distributions.

There is an alternative approach based on the concept of adjoint operators. The adjoint of $I^{\alpha}$ is an operator $K^{\alpha}$ defined by

$$
K^{\alpha} \phi(x)=\frac{1}{\Gamma(\alpha)} \int_{x}^{\infty}(u-x)^{\alpha-1} \phi(u) d u
$$

if $\operatorname{Re} \alpha>0$. Under certain conditions we have the formula for fractional integration by parts [7]

$$
\int_{0}^{\infty} I^{\alpha} f(x) \phi(x) d x=\int_{0}^{\infty} f(x) K^{\alpha} \phi(x) d x
$$

or

$$
\left(I^{\alpha} f, \phi\right)=\left(f, K^{\alpha} \phi\right)
$$

and this can be used to define $I^{\alpha}$ on a suitable class of generalised functions. The space of testing functions must be chosen so as to permit multiplication by fractional powers of the variable, and to admit the operator $K^{\alpha}$. 
This approach was indicated in an earlier paper [1] by the present author and A. C. McBride and it was carried through there for certain operators of fractional integration developed, for ordinary functions, by H. Kober [3]. Since [1] was written, Mr. McBride has further developed the theory of Kober's operators on certain classes of generalised functions and has also made some applications of it.

In Kober's operators, integration and multiplication by powers of $x$ are balanced in such a manner as to make the operator turn a function that belongs to $L_{p}(0, \infty)$ into another function of that class. The operator $I^{\alpha}$ behaves rather differently. For it, the behaviour at infinity is irrelevant. It is not necessary for $f$ to be in $L(0, \infty)$, and even if $f$ is integrable at infinity, $I^{\alpha} f$ will in general not have this property; while on the other hand $I^{\alpha} f$, for an integrable $f$, will be better than integrable at the origin.

Professor E. R. Love [6] has studied the operator $I^{\alpha}$ on classes $Q_{q}$ of functions $f$ for which $x^{q} f(x)$ is integrable on $[0, l]$ for each $l>0$. Clearly we must have $q \leqq 0$ for $I^{\alpha} f$ to exist. Love proves that for $f \in Q_{q}$ we have $I^{\alpha} f \in Q_{\varepsilon-\operatorname{Re} \alpha}$ for each $\varepsilon>0$ if $q=0$, and $I^{x} f \in Q_{q-\operatorname{Re} \alpha}$ if $q<0$. He has also given [5] corresponding results for $K^{\alpha}$. Furthermore, he made a very careful and detailed study of the first index law

$$
I^{\beta} I^{\alpha}=I^{\alpha+\beta}
$$

and of the second index law

$$
I^{\gamma} x^{\beta} I^{\alpha}=x^{-\alpha} I^{-\beta} x^{-\gamma} \quad \alpha+\beta+\gamma=0
$$

on the classes $Q_{q}$.

The aim of this paper is to extend these results to corresponding classes of generalised functions. One hopes to be able to dispense with the many case distinctions that are necessary in $Q_{q}$. As generalised functions are differentiable, $I^{\alpha} f$ can be defined for all $\alpha$, and there is no need to make a distinction according as $\operatorname{Re} \alpha>0,=0$, or $<0$. Moreover, many of the technical steps are performed on testing functions that are infinitely differentiable and have compact support; they require much less justification. Also, the operator $I^{\alpha}$ is continuous, and so certain inequalities are automatically indicated; these inequalities could be but have not been proved for functions. However, some of the results of the theory in $Q_{q}$, often precisely those requiring the most perspicacious analysis, cannot be expected to be included in the study of spaces of generalised functions. For instance, for $f \in Q_{q}, I^{\alpha} f$ exists for arbitrary $\alpha$ as a generalised function if $q<0$, and $I^{\alpha} f$ belongs to that space of generalised functions containing $Q_{q-\operatorname{Re} \alpha}$, but it does not follow that in case also $\operatorname{Re} \alpha>0, I^{\alpha} f$ exists as a function and belongs to $Q_{q-\operatorname{Re} \alpha}$. Thus, the present results, while they go further in some directions than Love's, do not include the latter. 
For each real $p$ and positive $l$, let $\mathscr{I}_{p l}$ be the collection of all those complex valued infinitely differentiable functions $\phi$ of a positive real variable $x$ which vanish outside the interval $[0, l]$ and for which, for each non-negative integer $k$,

$$
\gamma_{p k}(\phi)=\sup \left\{x^{-p+k}\left|\phi^{(k)}(x)\right|: x>0\right\}
$$

is finite. As in the case $p=0[1], \mathscr{I}_{p l}$ endowed with the family $\left\{\gamma_{p k}: k=0,1,2, \cdots\right\}$ of seminorms is a complete countably multinormed space, and for fixed real $p$

$$
\mathscr{I}_{p}=\bigcup_{i=1}^{\infty} \mathscr{I}_{p l}
$$

is a complete strict countable union space. Also, in Zemanian's notation [8, section 4.2], $\mathscr{I} \subset \mathscr{M}_{a, b}$ for $a \leqq p+1$ and any $b$.

If $p<q, \mathscr{I}_{p} \supset \mathscr{I}_{q}$ and the injection of $\mathscr{I}_{q}$ into $\mathscr{I}_{p}$ is continuous.

Let $\alpha$ be an arbitrary complex number and $a=\operatorname{Re} \alpha$. The operator $x^{\alpha}$ is defined on $\mathscr{I}_{p}$ by

$$
\left(x^{\alpha} \phi\right)(x)=x^{\alpha} \phi(x) .
$$

One proves as in [1] that $x^{\alpha}$ maps $\mathscr{I}_{p}$ continuously into $\mathscr{I}_{q}$ for any $q$ satisfying $q \leqq p+a$. The mapping is onto, and in fact an isomorphism, if $q=p+a$.

The operator $D$ is defined by

$$
(D \phi)(x)=\phi^{\prime}(x)
$$

and, as $\gamma_{q k}\left(\phi^{\prime}\right)=\gamma_{q+1, k+1}(\phi)$, it maps $\mathscr{I}_{p}$ continuously into $\mathscr{I}_{q}$ with $q \leqq p-1$. A counterexample given in [1] can be adapted to show that the mapping is not onto, not even for $q=p-1$.

For $\phi \in \mathscr{I}_{p l}$ for some real $p$ and positive $l$ and $a=\operatorname{Re} \alpha>0$ define $K^{\alpha} \phi$ by (1.2). If

$$
K^{\alpha+1} \phi^{\prime}(x)=\frac{1}{\Gamma(\alpha+1)} \int_{x}^{\infty}(u-x)^{\alpha} \phi^{\prime}(u) d u
$$

is integrated by parts, the integrated terms vanish and we have

$$
K^{\alpha+1} \phi^{\prime}=-K^{\alpha} \phi .
$$

This makes it possible to extend the definition of $K^{\alpha} \phi$ to all $\alpha$. For $n=0,1,2, \cdots$ and $\operatorname{Re} \alpha>-n$, let

$$
K^{\alpha} \phi=(-1)^{n} K^{\alpha+n} \phi^{(n)} .
$$

For fixed $x, K^{\alpha} \phi(x)$ is an entire function of $\alpha$. One also has

$$
\begin{aligned}
D^{k} K^{\alpha} \phi & =(-1)^{n} K^{\alpha+n} \phi^{(k+n)} \\
& =(-1)^{k} K^{\alpha-n} \phi=K^{\alpha} D^{k} \phi \quad \operatorname{Re} \alpha>-n .
\end{aligned}
$$


For $\phi \in \mathscr{I}_{p l}, K^{\alpha} \phi$ is clearly infinitely differentiable for positive $x$, and vanishes when $x>l$. Furthermore,

and so

$$
D^{k} K^{\alpha} \phi(x)=\frac{(-1)^{n}}{\Gamma(\alpha+n)} \int_{x}^{\infty}(u-x)^{x+n-1} \phi^{(k+n)}(u) d u
$$

if $0<x<l$.

$$
\left|D^{k} K^{\alpha} \phi(x)\right| \leqq \frac{\gamma_{p, k+n}(\phi)}{|\Gamma(\alpha+n)|} \int_{x}^{l}(u-x)^{a+n-1} u^{p-k-n} d u
$$

As $x \rightarrow 0+$,

$$
\begin{aligned}
\int_{x}^{l}(u-x)^{a+n-1} u^{p-k-n} d u & =x^{a+p-k} \int_{1}^{l / x}(t-1)^{a+n-1} t^{p-k-n} d t \\
& =O\left(x^{p+a-k}\right) \text { if } p+a-k<0 \\
& =O\left(\log \frac{1}{x}\right) \quad \text { if } p+a-k=0 \\
& =O(1) \quad \text { if } p+a-k>0
\end{aligned}
$$

Consequently, $\gamma_{q k}\left(K^{\alpha} \phi\right)<\infty$ for all $k$ and $\phi \in \mathscr{I}_{p l}$ provided either $q \leqq p+a<0$ or $q \leqq 0<p+a$ or else $q<0$ and $p+a=0$.

We thus see that $K^{\alpha}$ is a continuous map of $\mathscr{I}_{p}$ into $\mathscr{I}_{p+a}$ if $p+a<0$, into $\mathscr{I}_{0}$ if $p+a>0$, and into $\mathscr{I}_{-\varepsilon}$ for each $\varepsilon>0$ if $p+a=0$.

It follows from (2.3) that

and so

$$
K_{0} \phi(x)=-K^{1} \phi^{\prime}(x)=-\int_{x}^{\infty} \phi^{\prime}(u) d u
$$

$$
K_{0} \phi=\phi .
$$

Next, the first index law for $K^{\alpha}$,

$$
K^{\alpha} K^{\beta} \phi=K^{\alpha+\beta} \phi
$$

can be proved for any $\left.\phi \in C^{\infty}\right] 0, \infty[$ vanishing outside of a compact interval and for $\operatorname{Re} \alpha>0, \operatorname{Re} \beta>0$ by writing the left hand side as a repeated integral and interchanging the order of integrations. This relation can then be extended to all $\alpha$ and $\beta$ either by analytic continuation or by employing (2.3) and (2.4).

From (2.5) and (2.6),

$$
K^{-\alpha} K^{\alpha} \phi=\phi
$$

for $\phi \in \mathscr{I}_{p}$.

We now turn to what is in effect Kober's operators. Let $\rho, \sigma, \tau$ be complex numbers satisfying $\rho+\sigma+\tau=0$, set $\operatorname{Re} \rho=r, \operatorname{Re} \sigma=s, \operatorname{Re} \tau=t$, and consider the operators 


$$
K_{\rho \sigma \tau}=x^{\tau} K^{\sigma} x^{\rho} .
$$

$K_{\rho \sigma \tau}$ maps $\mathscr{I}_{p}$ into itself if $p<t$, into $\mathscr{I}_{t}$ if $p>t$, and into $\mathscr{I}_{t-\varepsilon}$ for each $\varepsilon>0$ if $p=t$.

Kober's operators commute. This was shown in $L_{p}(0, \infty)$ by Kober [3, theorem 4, second part] and in classes similar to $Q_{q}$ by Love [5, theorem 3]. For the sake of completeness we reproduce the proof here especially since the justification of the formal operations is easier since all integrals are over a finite range.

Let $\lambda, \mu, v, \rho, \sigma, \tau$ be complex numbers with $\lambda+\mu+v=\rho+\sigma+\tau=0$. It is sufficient to prove

$$
K_{\lambda \sigma \tau} K_{\gamma \mu \nu} \phi=K_{\lambda \mu v} K_{\rho \sigma \tau} \phi \quad \phi \in \mathscr{I}_{p}
$$

for $\operatorname{Re} \sigma>0, \operatorname{Re} \mu>0$; the extension to all $\sigma, \mu$ follows by analytic continuation.

Now

$$
\begin{aligned}
K_{\rho \sigma \tau} K_{\lambda \mu \nu} \phi(x) & \\
& =\int_{x}^{\infty} x^{\tau} \frac{(u-x)^{\sigma-1}}{\Gamma(\sigma)} u^{\rho}\left(\int_{u}^{\infty} u^{v} \frac{(v-u)^{\mu-1}}{\Gamma(\mu)} v^{\lambda} \phi(v) d v\right) d u \\
& =\int_{x}^{\infty} A(\lambda, \mu, v ; \rho, \sigma, \tau ; v, x) \phi(v) d v
\end{aligned}
$$

where

$$
\begin{aligned}
& \Gamma(\sigma) \Gamma(\mu) A(\lambda, \mu, v ; \rho, \sigma, \tau ; v, x) \\
& \quad=\int_{x}^{v} v^{\lambda}(v-u)^{\mu-1} u^{\nu+\rho}(u-x)^{\sigma-1} x^{\tau} d u .
\end{aligned}
$$

Making a change of variable, $u=v x / w$, the last integral becomes

$$
\int_{x}^{v} v^{\lambda+\mu+v+\rho}(v-w)^{\sigma-1} w^{-\mu-v-\rho-\sigma}(w-x)^{\mu-1} x^{\nu+\rho+\sigma+\tau} d w
$$

and in view of $\lambda+\mu+v=\rho+\sigma+\tau=0$ this is

$$
\Gamma(\sigma) \Gamma(\mu) A(\rho, \sigma, \tau ; \lambda, \mu, v ; v, x) .
$$

The symmetry of $A$ in the triplets $\lambda, \mu, v$ and $\rho, \sigma, \tau$ proves (2.9).

The second index law for $K^{\alpha}$,

$$
K^{\alpha} x^{\beta} K^{\gamma} \phi=x^{-\gamma} K^{-\beta} x^{-\alpha} \phi, \alpha+\beta+\gamma=0, \quad \phi \in \mathscr{I}_{p}
$$

follows from (2.6) and (2.9). 


$$
\begin{aligned}
K^{\alpha} x^{\beta} K^{\gamma} \phi & =K_{-\alpha, \alpha, 0} K_{0, \gamma-\gamma} \phi \\
& =K_{0, \gamma-\gamma} K_{-\alpha \alpha, 0} \phi \\
& =x^{-\gamma} K^{\gamma} K^{\alpha} x^{-\alpha} \phi \\
& =x^{-\gamma} K^{\alpha+\gamma} x^{-\gamma} \phi=x^{-\gamma} K^{-\beta} x^{-\alpha} \phi .
\end{aligned}
$$

We now turn to the space $\mathscr{I}_{p}^{\prime}$ of generalised functions, i.e., the continuous linear functionals on $\mathscr{I}_{p}$. Love's class $Q_{p}$ can be embedded in $\mathscr{I}_{p}^{\prime}$. Each $f$ in $Q_{p}$ defines a linear functional on $\mathscr{I}_{p}$ by the definition

$$
(f, \phi)=\int_{0}^{\infty} f(x) \phi(x) d x .
$$

The integral exists as a Lebesgue integral over a finite interval. If $\phi \in \mathscr{I}_{p l}$, we have

$$
(f, \phi) \leqq \gamma_{p, 0}(\phi) \int_{0}^{l} x^{p}|f(x)| d x
$$

and so the functional generated by $f$ which we shall also denote by $f$ is continuous.

If $p<q, \mathscr{I}_{p}^{\prime} \subset \mathscr{I}_{q}^{\prime}$, and the injection of $\mathscr{I}_{p}^{\prime}$ into $\mathscr{I}_{q}^{\prime}$ which assigns to $f \in \mathscr{I}_{p}^{\prime}$ the restriction of $f$ to $\mathscr{I}_{q}$ is continuous. We also denote by $\tilde{I}_{p}^{\prime}$ the collection of those generalised functions which belong to $\mathscr{I}_{q}^{\prime}$ for each $q>p$. Clearly $\mathscr{I}_{p}^{\prime} \subset \tilde{\mathscr{I}}_{p}^{\prime}$, and the example $f(x)=x^{-p} \log (1 / x)$ shows that $\mathscr{I}_{p}^{\prime} \neq \tilde{\mathscr{I}}_{p}^{\prime}$.

The operators $x^{\alpha}, D$, and $I^{\alpha}$ can be defined for our generalised functions by

$$
\begin{aligned}
& \left(x^{\alpha} f, \phi\right)=\left(f, x^{\alpha} \phi\right) \\
& (D f, \phi)=-(f, D \phi) \\
& \left(I^{\alpha} f, \phi\right)=\left(f, K^{\alpha} \phi\right) .
\end{aligned}
$$

It follows from the results of section 2 on the operators $x^{\alpha}, D, K^{\alpha}$ on $\mathscr{I}_{p}$, that $x^{\alpha}$ is an isomorphism of $\mathscr{I}_{p}^{\prime}$ onto $\mathscr{I}_{p-a}^{\prime}$ for any $p ; D$ is a continuous map of $\mathscr{I}_{p}^{\prime}$ into $\mathscr{I}_{p+1}^{\prime}$ for any $p$; and that $I^{\alpha}$ can be defined on $\mathscr{I}_{p}^{\prime}$ only if $p \leqq 0$, and then maps $\mathscr{I}_{p}^{\prime}$ into $\mathscr{I}_{p-a}^{\prime}$ if $p<0$ into $\tilde{\mathscr{I}}_{-a}^{\prime}$ if $p=0$. Moreover, $x^{\alpha}$ is also an algebraic isomorphism of $\tilde{\mathscr{I}}_{p}^{\prime}$ onto $\tilde{\mathscr{I}}_{p-a}^{\prime}$ for any $p ; D$ is a map of $\tilde{\mathscr{I}}_{p}^{\prime}$ into $\tilde{\mathscr{I}}_{p+1}^{\prime}$ for any $p$; and $I^{\alpha}$ a map of $\tilde{\mathscr{I}}_{p}^{\prime}$ into $\tilde{\mathscr{I}}_{p-a}^{\prime}$ for $p \leqq 0$.

The first index law can now be stated as

$$
I^{\beta} I^{\alpha} f=I^{\alpha+\beta} f .
$$

It holds for all $\alpha$ and $\beta$, with $a=\operatorname{Re} \alpha, b=\operatorname{Re} \beta$, if either (i) $f \in \mathscr{I}_{p}^{\prime}$ and $p<\min (0, a)$ or (ii) $f \in \tilde{I}_{p}^{\prime}$ and $p \leqq \min (0, a)$. In case (i), both sides of (3.5) are in $\mathscr{I}_{p-a-b}^{\prime}$, in case (ii) they are in $\tilde{\mathscr{I}}_{p-a-b}^{\prime}$. This follows from (2.6) in conjunction with the properties of $K^{\alpha}$. 
Love [6] has analysed (3.5) in detail when $f \in Q_{q}$, and it is of interest to see how far his results can be subsumed under (3.5). Of course, only conditions on $\alpha, \beta, f$ can be compared. Love proves that the operations on his functions result again in functions, and can assign these functions to certain $Q_{q}$ classes, while (3.5) assert the existence and equality of the two sides only in the sense of generalized functions.

In his theorem 1 , Love assumes $a>0, b>0, f \in Q_{0}$. As $Q_{0}$ can be embedded in $\mathscr{I}_{0}^{\prime}$, this accords well with the condition $p \leqq \min (0, a)$. Although he does not state it, it follows from his analysis that both sides of (3.5) are in $Q_{\varepsilon-a-b}$ for each $\varepsilon>0$, and that both sides are in $Q_{q-a-b}$ if $q<0$ and $f \in Q_{q}$. This is again in accordance with the statement that both sides of (3.5) are in $\mathscr{I}_{p \rightarrow a-b}^{\prime}$ if $p<\min (0, a)$ and in $\tilde{\mathscr{F}}_{p-a-b}^{\prime}$ if $p=\min (0, a)$.

The condition $b>0$ is required in Love's work to assure that both sides are functions that can be formed by using (1.1) as the definition of fractional integrals.

In theorem 2, Love assumes $a \leqq 0, b>0, I^{x} f$ exists in $Q_{0}$. Under these assumptions $f=I^{-\alpha}\left(I^{\alpha} f\right) \in \tilde{\mathscr{I}}_{a}^{\prime}$ and again $p=a \leqq \min (0, a)$. In a similar manner in the two remaining cases of Love's theorems 3 and 4 it can be verified that his conditions on $f$ are in agreement with (3.5).

Next, we define the operator

$$
I_{\alpha \beta \gamma}=x^{\alpha} I^{\beta} x^{\gamma}, \quad \alpha+\beta+\gamma=0
$$

and find that it maps $\mathscr{I}_{p}^{\prime}$ into itself if $p<\operatorname{Re} \gamma$ and maps $\mathscr{I}_{p}^{\prime}$ into $\tilde{\mathscr{I}}_{p}^{\prime}$ (and $\tilde{\mathscr{I}}_{p}^{\prime}$ into itself) if $p \leqq \operatorname{Re} \gamma$. If $\lambda+\mu+\nu=\rho+\sigma+\tau=0$, we have

$$
I_{\lambda \mu \nu} I_{\rho \sigma \mathrm{r}} f=I_{\rho \sigma \tau} I_{\lambda \mu \nu} f
$$

if either (i) $f \in \mathscr{I}_{p}^{\prime}, \quad p<\operatorname{Re} v$ and $p<\operatorname{Re} \tau$ when both sides are in $\mathscr{I}_{p}^{\prime}$, or (ii) $f \in \tilde{\mathscr{I}}_{p}^{\prime}, p \leqq \operatorname{Re} v, p \leqq \operatorname{Re} \tau$ when both sides are in $\tilde{\mathscr{I}}_{p}^{\prime}$. The proof follows from (2.9).

Either (3.7) or (2.10) can be used to prove the second index law in the form

$$
I^{\gamma} x^{\beta} I^{\alpha} f(x)=x^{-\alpha} I^{-\beta} x^{-\gamma} f(x) \quad \alpha+\beta+\gamma=0
$$

valid if either (i) $f \in \mathscr{I}_{p}^{\prime}, p<\min (0,-c)$, when both sides are again in $\mathscr{I}_{p}^{\prime}$ or (ii) $f \in \tilde{\mathscr{I}}_{p}^{\prime}, p \leqq \min (0,-c)$, when both sides are in $\tilde{\mathscr{I}_{p}^{\prime}}$. Here $c=\operatorname{Re} \gamma$.

Again a comparison with Love's results is instructive. In theorem 5 of [6] Love assumes $a>0, b<0, c>0, f \in Q_{-c}$ and shows that (3.8) holds a.e., with both sides in $Q_{\varepsilon-c}$ for each $\varepsilon>0$. This corresponds to $p=-c$ and agrees with our results. The conditions $a>0, b<0$ which do not appear in our work are needed to insure the existence of $I^{\alpha} f$ and $I^{-\beta} c^{-\gamma} f$ as integrals. Love also proves that in case also $f \in Q_{-c-\eta}$ then both sides of (3.8) are again in $Q_{-c-\eta}$. This is again in agreement with our work, as $p=-c-\eta<\min (0,-c)$. It can be seen similarly that Love's theorem 6 is in agreement with our results. 
In his theorem 7 Love assumes $a<0, b<0, c>0$ and also that $I^{\alpha} f$ exists and belongs to $Q_{b}$ (and hence to $\mathscr{I}_{b}^{\prime}$ ). It follows that $f=I^{-\alpha}\left(I^{\alpha} f\right) \in \mathscr{I}_{a+b}^{\prime}=\mathscr{I}_{-c}^{\prime}$ and hence (3.8) holds with both sides in $\tilde{\mathscr{I}}_{-c}^{\prime}$. The conditions $a<0, b<0$ are not needed for the validity of (3.8) in $\tilde{\mathscr{I}}_{-c}^{\prime}$ : they enable Love to prove that actually both sides exist as functions which are in $Q_{\varepsilon-c}$ for each $\varepsilon>0$. The further result that if $I^{\alpha} f \in Q_{b-\eta}$ with $\eta>0$ then both sides of (3.8) are in $Q_{b-\eta}$ is also in agreement with our results.

In a similar manner it can be verified that Love's theorems $8-10$ are also in agreement with the results presented here. In each case, some of Love's assumptions insure that $p \leqq \min (0,-c)$, while others, not appearing above, are needed to insure that both sides of (3.8) exist as functions in certain $Q_{q}$ classes.

\section{Acknowledgement}

This paper was written while I was a Visiting Professor at the University of Melbourne and owes much to Professor Love's work on fractional integrals. I should like to express my thanks to Melbourne University for their hospitality and to Professor Love for stimulating discussions, and also for allowing me to read his unpublished papers.

Note added on 17th May, 1972

A. E.

Additional material on fractional integrals of generalised functions will be found in: A. C. McBride "A theory of fractional integration for generalised functions with applications", Ph.D. Thesis, Edinburgh, 1971.

\section{References}

[1] A. Erdélyi and A. C. McBride, 'Fractional integrals of distributions', SIAM J. on Math. Analysis (1970) 547-557.

[2] I. M. Gelfand and G. E. Shilov, Generalized functions Vol. 1 (Academic Press, New York, 1964).

[3] H. Kober, 'On fractional integrals and derivatives', Quart. J. of Math. (Oxford) 11 (1940), 193-211.

[4] E. R. Love, 'Some integral equations involving hypergeometric functions', Proc. Edinburgh Math. Soc. (2) 15 (1967), 169-198.

[5] E. R. Love, 'Two more hypergeometric integral equations', Proc. Cambridge Philos. Soc. 63 (1967), 1055-1076.

[6] E. R. Love, 'Two index laws for fractional integrals and derivatives', to appear in the $J$. of Australian Math. Soc.

[7] E. R. Love and L. C. Young, 'On fractional integration by parts', Proc. London Math. Soc. (2) 44 (1938), 1-28.

[8] A. H. Zemanian, Generalized integral transformations (Interscience Publishers, New York, 1968).

University of Edinburgh and

University of Melbourne 